\title{
画HAD
}

DOI: http://doi.org/10.22585/hospdomic.v3i3.75

\section{Derivación directa de urgencias a hospitalización a domicilio en descompensación aguda de insuficiencia cardiaca crónica}

\section{Direct Admission to Hospital at Home from Emergency Room for Acutely Decompensated Chronic Heart Filure}

José Regalado De Los Cobos', Miriam Delgado Vicente', Jose Medrano Laporte', Montserrat Gómez Rodríguez de Mendarozqueta', Esther Oceja Barrutieta', María De Juan Rodríguez'

1. Servicio de Hospitalización a Domicilio de Vitoria-Gasteiz, España.

Correspondencia/Correspondence

José Regalado De Los Cobos

Unidad de Hospitalización a Domicilio. Hospital

Universitario de Alava- Txagorritxu.

c/ José Atxotegi n 3. 01009 - VITORIA-

GASTEIZ - SPAIN

jose.regaladodeloscobos@osakidetza.eus

Recibido/Received

21.05.2019

Aceptado/Accepted

13.06.2019
Conflicto de Intereses/Competing interest Los autores y autoras no declaran ningún tipo de conflicto de interes.

Agradecimientos/Acknowledgments

No declarados.

CÓMO CITAR ESTE TRABAJO | HOW TO CITE THIS PAPER

Regalado J, Delgado M, Medrano J, Gómez M, Oceja E, De Juan M, Apraiz L, Frago I. Derivación directa de urgencias a hospitalización a domicilio en descompensación aguda de insuficiencia cardiaca crónica. Hosp Domic. 2019;3(3):193-201 


\section{RESUMEN}

Objetivo: Evaluar la severidad clínica en una serie histórica de pacientes atendidos en Hospitalización a Domicilio (HaD) por descompensación aguda de insuficiencia cardiaca crónica (DAIC) remitidos directamente desde el Servicio de Urgencias y la influencia en el resultado clínico.

Método: Análisis retrospectivo de casos enviados desde el Servicio de Urgencias a $\mathrm{HaD}$ de Vitoria-Gasteiz de 2006 a 2016. Se registró el número de ingresos por DAIC en año anterior y puntuación en escala EFFECT. Se analizó la influencia de estas variables en el reenvío de casos al hospital para ingreso convencional.

Resultados: De 214 casos analizados 16.8\% fueron reenviados al hospital. La combinación de la puntuación EFFECT y el número de hospitalizaciones por DAIC en el año precedente configura dos grupos de pacientes con riesgo de reenvío al hospital de $9,1 \%$ y $29,6 \%$ (R.R.= 3,26, I.C. = 1,73-6,15).

Conclusiones: H a D trata eficazmente a pacientes remitidos directamente desde el Servicio de Urgencias con descompensación de insuficiencia cardiaca crónica con diferentes severidades.

La escala EFFECT y las hospitalizaciones previas por DAIC son útiles para predecir el riesgo de reenvío al hospital desde $\mathrm{H}$ a $\mathrm{D}$ y por lo tanto constituyen una ayuda a la hora de decidir en el Servicio de Urgencias qué pacientes derivar a had y cuáles ingresar en planta.

Palabras clave: Servicios de Atención de Salud a Domicilio; Servicios de Atención a Domicilio Provisto por Hospital; Insuficiencia cardiaca; Escala predictiva EFFECT.

\section{ABSTRACT}

Aim: To assess the clinical severity of an historical series of patients directly sent from the Emergency Room to Hospital at Home for treatment of Acutely Decompensated Chronic Heart Failure (ADCHF) and how it influences the clinical result.

Methods: Retrospective analysis of patients referred directly from the Emergency Department to Hospital at Home from 2006 to 2016. The number of admissions due to ADCHF during the year before and the score at the EFFECT predictive scale at presentation were recorded and their influence in the need to return to hospital was assessed.

Results: 214 cases were evaluated, $16.8 \%$ of them needed to return to hospital. Using the EFFECT score and the number of previous admissions the patients could be divided into two groups with low (9.1\%) and high (29.6\%) return to hospital rates (R.R. = 3.26; C.I. = 1.73-6.15).

Conclusions: Patients with acutely decompensated chronic heart failure referred directly from the Emergency Department can be effectively treated in Hospital at Home.

The score at the EFFECT predictive tool and the number of previous admissions are useful to estimate the risk of sending the patient back to hospital and therefore can be used at the Emergency Room as a help to select patients for $\mathrm{H}$ a $\mathrm{H}$.

Keywords: Home Care Services; Home Care Services, Hospital-Based; Heart Failure; EFFECT score. 


\section{INTRODUCCIÓN}

La insuficiencia cardiaca (IC) es el principal motivo no quirúrgico de ingreso hospitalario para los mayores de 65 años en España (1). En el hospital Universitario de Alava, que atiende a una población de 300.000 habitantes, la IC motiva una media de 1000 ingresos hospitalarios anuales. Los ingresos hospitalarios suponen el $75 \%$ del coste económico directo que comporta la asistencia a esta enfermedad que se ha estimado en un 2,5-4\% del gasto sanitario total (2). Es previsible que el número de ingresos vaya en aumento debido a que la prevalencia de la IC es creciente con la edad ( $8 \%$ en la población de edad entre 65 y 74 años, 16,1\% entre los mayores de 74) (3). Y, por otro lado, esta última franja de edad presenta en nuestro entorno un crecimiento exponencial en las últimas décadas (4), que se mantendrá previsiblemente hasta 2050, cuando cumplan esa edad los últimos nacidos durante el "baby boom" Debe tenerse en consideración también que las hospitalizaciones inciden negativamente tanto en el pronóstico (5) como en la calidad de vida de los pacientes (6). Por todo ello resulta de interés disminuir la frecuencia de hospitalizaciones por insuficiencia cardiaca y existen diferentes vías para conseguirlo (7). Sin embargo este objetivo se resiste a ser alcanzado, a diferencia de lo que ha ocurrido en otras patologías y a pesar de disponer de diferentes herramientas de estratificación de pronóstico que ayude a decidir si el paciente puede ser dado de alta con control ambulatorio (8). La derivación directa desde el Servicio de Urgencia (SU) a Hospitalización a Domicilio (HaD) es una opción que ha demostrado ser una forma eficaz y segura de evitar ingresos hospitalarios indicados en numerosas situaciones (9), también en la descompensación aguda de insuficiencia cardiaca crónica (DAICC) como dos estudios randomizados han demostrado $(10,11)$. Sin embargo, estos estudios incluyen un número de casos pequeño y que representa una proporción muy baja con respecto al total de pacientes atendidos por el hospital en el mismo período. Así mismo, proporcionan poca información acerca de la severidad de los casos incluidos. Quizás se reclutaron casos con muy baja severidad.

El propósito del presente estudio es valorar la severidad de los casos atendidos por DAICC en una Unidad de HaD, compararla con una serie de pacientes hospitalizados y evaluar el impacto que el nivel de severidad provoca en la eficacia de la asistencia en HaD.

\section{MÉTODOS}

Estudio retrospectivo de los casos derivados desde el SU a la Unidad de HaD del Hospital Universitario Vitoria-Gasteiz para tratamiento por agudización de insuficencia cardiaca crónica, excluidos los pacientes en situación de cuidados paliativos, desde Enero de 2006 hasta Junio de 2016. En el SU además de establecer el diagnóstico e iniciar el tratamiento, antes de derivar un caso a $\mathrm{HaD}$, se descartan los criterios de exclusión que se detallan en la Tabla 1.

En el estudio retrospectivo se registraron para cada caso:

1. Número de ingresos por DAICC en los 12 meses previos.

2. Puntuación en la escala EFFECT, que predice la mortalidad a 12 meses a partir de 11 variables clínicas que presenta el paciente en el momento de consultar por descompensación y son fácilmente obtenibles a partir del informe de asistencia en el Servicio de Urgencias: edad; antecedentes de demencia, enfermedad cerebrovascular, EPOC, cáncer, cirrosis; presión arterial, frecuencia respiratoria; anemia, hiponatremia y nivel de urea (12). La escala se describió en 2003 a partir de una cohorte de 2624 pacientes ingresados por insuficiencia cardiaca en diferentes hospitales de Canadá entre 1999 y 2001. La división en cinco quintiles correspondió 
con los siguientes puntos de corte: <80, 80-93, 94-107, 108-123 y >123 puntos y la mortalidad a un año asociada a cada uno de ellos fue de 9\%; 17,7\%; 29,1\%; 42,1\% y 66,3\% respectivamente.

3. Destino al alta de HaD: Atención Primaria o retorno al hospital.

4. Mortalidad por cualquier causa en el plazo de un año posterior al ingreso en HaD.

Tabla 1. Criterios de exclusión para derivar pacientes a HaD

\begin{tabular}{|l|l|}
\hline GENERALES & $\begin{array}{l}\text { Carecer de domicilio en la zona atendida con condiciones adecuadas de higiene y } \\
\text { seguridad. } \\
\text { Carecer de ayuda familiar o contratada. } \\
\text { Carecer de teléfono. }\end{array}$ \\
\hline ESPECÍFICOS & $\begin{array}{l}\text { Etiología de cardiopatía no conocida. } \\
\text { Síndrome coronario agudo. } \\
\text { Arritmia no controlada. } \\
\text { Riesgo de muerte súbita, necesidad de monitorización ECG o Medicina Intensiva. } \\
\text { Necesidad o realización reciente de cirugía o intervencionismo cardiacos. } \\
\text { Portadores de marcapaso o DAl o DAl-TRC que requieran revisión del dispositivo. } \\
\text { Descompensación leve controlable de forma ambulatoria }\end{array}$ \\
\hline
\end{tabular}

Comparación con otras series: se compara la serie actual con la serie a partir de la cual se derivó la herramienta predictiva EFFECT (12) y con una serie de pacientes ingresados por insuficiencia cardiaca en 2011 en el Hospital Santiago Apóstol de Vitoria-Gasteiz (13).

\section{Análisis estadístico}

Para valorar la relación entre las variables independientes (Ingresos en año previo y puntuación EFFECT) y la variable de resultado principal, siendo esta una variable binaria (alta / retorno), se ha realizado una regresión logística, con análisis univariable y multivariable, estimando en ambos casos el valor de Akaike Informatio Criterion (AIC).

\section{RESULTADOS}

En el período estudiado HaD recibió del SU 214 casos de DAICC. Se analizaron 101 varones y 113 mujeres, de edad media 79,7 ( $\mathrm{DE}=7,6)$ y $84,6(\mathrm{DE}=7)$ respectivamente. Durante el tratamiento en $\mathrm{HaD}$ no falleció ningún paciente. 36 casos (16.8\%) fueron reenviados para ingreso hospitalario mientras que los 178 restantes fueron dados de alta a Atención Primaria.

Comparación de la serie con otras series de pacientes hospitalizados por insuficiencia cardiaca.

En la Tabla 2 se muestra la distribución de casos de la serie actual en los cinco grupos de riesgo establecidos de acuerdo con los puntos de corte antes descritos y la mortalidad a 1 año en cada uno de ellos. Se muestran los mismos datos correspondientes a las series de comparación $(12,13)$. 
Tabla 2. Comparación de serie actual con serie histórica de pacientes hospitalizados por insuficiencia cardiaca

\begin{tabular}{|l|l|l|l|l|l|l|}
\hline & \multicolumn{2}{|c|}{$\%$ de casos en la serie } & \multicolumn{2}{l|}{ Mortalidad a 1 año } & \\
\hline $\begin{array}{l}\text { Puntuación } \\
\text { EFFECT }\end{array}$ & $\begin{array}{l}\text { Actual } \\
(\mathbf{n}=\mathbf{2 1 4})\end{array}$ & $\begin{array}{l}\text { Lee } \\
(\mathbf{n}=\mathbf{2 6 2 4})\end{array}$ & $\begin{array}{l}\text { H.S.A. } \\
(\mathbf{n}=\mathbf{2 2 7})\end{array}$ & $\begin{array}{l}\text { Actual * } \\
(\mathbf{n = 1 7 7 )}\end{array}$ & $\begin{array}{l}\text { Lee } \\
(\mathbf{n = 2 6 2 4})\end{array}$ & $\begin{array}{l}\text { H.S.A. * } \\
(\mathbf{n}=\mathbf{1 7 5})\end{array}$ \\
\hline$<80$ & $15.9 \%$ & $20 \%$ & $8.7 \%$ & 6.3 & $9 \%$ & $5.3 \%$ \\
\hline $80-93$ & $23.4 \%$ & $20 \%$ & $12.6 \%$ & $10 \%$ & $17,7 \%$ & $12 \%$ \\
\hline $94-107$ & $28.5 \%$ & $20 \%$ & $22.2 \%$ & $36.5 \%$ & $29,1 \%$ & $19.5 \%$ \\
\hline $108-123$ & $18.7 \%$ & $20 \%$ & $17 \%$ & $45.5 \%$ & $42,1 \%$ & $55.2 \%$ \\
\hline$>123$ & $13,6 \%$ & $20 \%$ & $39.6 \%$ & $40 \%$ & $66,3 \%$ & $55.7 \%$ \\
\hline GLOBAL & $100 \%$ & $100 \%$ & $100 \%$ & $27.1 \%$ & $32.9 \%$ & $35.4 \%$ \\
\hline
\end{tabular}

*La mortalidad se analizó en los casos en que se pudo completar el seguimiento de 12 meses y, en los pacientes con más de un episodio de atención por IC en el período estudiado, sólo se tomó para el análisis el primero de ellos.

\section{Retorno al hospital y puntuación EFFECT}

La distribución de los casos en los cinco niveles de puntuación EFFECT fue la siguiente: < 80 puntos: 34 casos; 80-93: 50; 94-107: 61; 108-123: 40 y, finalmente, >123: 29 casos.

La proporción casos reenviados al hospital en cada uno de esos cinco grupos fue respectivamente: $8,8 \%, 10 \%, 18,3 \%, 20 \%$ y $31 \%$. Por lo tanto, se pueden reagrupar los cinco grupos en tres: en los casos con puntuación <94, el reenvío ocurrió en 9,5\%. Puntuación = 94-123 se asoció con un porcentaje de reenvío de 19\%.(RR =1,92). Finalmente, puntuación > 123 implicó un porcentaje de reenvíos de $31 \%$. $(R R=3,26)$. En el análisis univariable, la puntuación EFFECT se muestra como factor de riesgo para el retorno $(p$ value $=0,00566, \mathrm{AIC}=190,14)$.

\section{Retorno al hospital y hospitalizaciones por IC en año precedente}

De los 214 casos, 113 no habían presentado ninguna hospitalización por IC en el año precedente, 43 casos habían presentado una y 57 más de una. (Falta registro en un caso). La proporción de reenvío al hospital fue respectivamente: $11 / 113=9,7 \% ; 9 / 43=20,9 \%(R R=2,15)$ y $16 / 57=28,1 \%$ $(R R=2,88)$. El análisis univariable muestra también significativa la relación con el reenvío ( $p$ value = 0,0205, $\mathrm{AIC}=192,43$ ). 


\section{Combinación de ambas variables}

Al realizar el análisis bivariable EFFECT se mantiene como factor de riesgo ( $p$ value $=0.0157$ ), pero no así los ingresos previos ( $p$ value $=0.0692$ ). Sin embargo el valor de AIC es mejor ( $m a ́ s$ bajo) para el modelo multivariable ( $\mathrm{AIC}=188.6)$, lo que indica que el modelo multivariable es mejor predictor que cada una de las dos variables individuales.

De acuerdo con ello, se ha categorizado la puntuación en la escala EFFECT, en 3 grupos: $<94$, 94-123 y >123. Y el número de ingresos por IC en año previo en dos: 0 y $>0$.

A la vista del porcentaje de reenvío en cada uno de los 6 subgrupos de pacientes que resultan del valor combinado de las dos variables, se propone una nueva escala de puntuación combinando ambas variables, resultado de la suma de los puntos asignados según el valor en cada una de las dos variables, tal y como muestra la Tabla 3. Esta escala permite definir dos grupos, correspondientes a las puntuaciones: $<2$ y $\geq 2$, cuyos porcentajes de reenvío se muestran en la Tabla 4.

\section{DISCUSIÓN}

El presente estudio pone de manifiesto en primer lugar que HaD trató de forma eficaz a un 83,2 $\%$ (178 de 214) de los pacientes con DAIC remitidos directamente desde el Servicio de Urgencias. La serie incluye casos de todos los niveles pronósticos establecidos por la escala predictiva EFFECT y el $32.3 \%$ del total pertenecen a los dos niveles de mayor severidad. Aunque la cifra es elevada, es inferior a la que presentan las otras dos series de pacientes hospitalizados por insuficiencia cardiaca, que son $40 \%$ y $56.6 \%$ respectivamente. En consonancia con ello, en la serie de $\mathrm{H}$ a D también la mortalidad a 1 año fue menor que en las otras dos (27.1\% frente a $32.9 \%$ y $35.4 \%$ ), (Tabla2).

En segundo lugar, de forma indirecta, el trabajo pone de manifiesto la pequeña magnitud de la derivación de casos de DAIC de Urgencias a H a D en nuestra Organización Sanitaria Integrada: 214 casos en 10,5 años, cuando se contabilizan unos 1000 ingresos hospitalarios anuales por insuficiencia cardiaca.

En tercer lugar, los resultados sugieren que un baremo sencillo construido a partir de la puntuación en la escala EFFECT y el número de ingresos por IC en el año previo, puede ser de utilidad para seleccionar pacientes con descompensación de IC candidatos a ser tratados en HaD con riesgo bajo (9.1\%) de necesitar ser reenviados al hospital para ingreso convencional. O también para identificar a otros pacientes que, por presentar un riesgo mayor de retorno al hospital (29.6\%), en caso de ser remitidos a H a D serían candidatos a un seguimiento más intensivo en este servicio.

La eficacia de $\mathrm{HaD}$, entendida como finalización de la asistencia sin necesidad de reenviar al paciente al hospital, se ve influida por tanto por la severidad de la descompensación. Se han descrito otros factores que predisponen al paciente al ingreso hospitalario pero no han sido analizados en el presente estudio. Por ejemplo, el estado funcional evaluado por medio del índice de Barthel, influencia el resultado terapéutico en ancianos tratados por insuficiencia cardiaca $(14,15)$ y también influencia la tasa de retorno tras alta al Servicio de Urgencias (16). La presencia de síntomas depresivos (17) y el nivel socioeconómico (18) influencian también las tasas de rehospitalización y la aceptación de la asistencia en $\mathrm{HaD}$ (19).

Una valoración completa en los Servicios de Urgencias de todos estos factores condicionantes, además de los propuestos en el presente trabajo, permitiría una mejor selección de candidatos para recibir tratamiento en HaD. Ahora bien, en los Servicios de Urgencias habitualmente no es fácil realizar valoraciones tan exaustivas. Valorar los dos factores que se proponen en el presente 
estudio resultaría más asequible y suficiente para aumentar el número de derivaciones que a día de hoy continúa siendo escaso.

El estudio presenta algunas limitaciones como que los datos están referidos a la actividad de una única Unidad de HaD y representan una proporción muy pequeña de los casos atendidos en los Servicios de Urgencias. Al tratarse de un análisis retrospectivo, pueden concurrir sesgos de selección no controlados. Sería necesario realizar una validación externa de la propuesta.

Como conclusiones, se puede afirmar que $\mathrm{HaD}$ resulta eficaz en el tratamiento de un elevado porcentaje de casos de DAICC, pero continua siendo infrautilizada. La existencia de ingresos previos y la puntuación en la escala EFFECT pueden ser una herramienta útil en los Servicios de Urgencias para seleccionar los casos que pueden ser derivados a $\mathrm{HaD}$ con mayor seguridad.

Tabla 3. Cálculo del índice combinado: EFFECT + Ingresos previos

\begin{tabular}{|l|l|}
\hline PUNTUACIÓN EFFECT & Puntos \\
\hline$<94$ & 0 \\
\hline $94-123$ & 1 \\
\hline$>123$ & 2 \\
\hline INGRESOS AÑO PREVIO & Puntos \\
\hline 0 & 0 \\
\hline$>0$ & 1 \\
\hline
\end{tabular}

Tabla 4. Retorno al hospital según puntuación en índice combinado

\begin{tabular}{|l|l|l|}
\hline Puntuación & Casos retornados (\%) & R.R. (I.C.) \\
\hline $0-1$ & $12 / 132(9.1 \%)$ & 1 \\
\hline $2-3$ & $24 / 81(29.6 \%)$ & $3.26(1.73-6.15)$ \\
\hline
\end{tabular}

\section{BIBLIOGRAFÍA}

1. Rodriguez-Artalejo F, Banegas Banegas JR, Guallar-Castillon P. Epidemiologia de la insuficiencia cardiaca. Rev Esp Cardiol. 2004;57(2):163-70. DOI: 10.1157/13057268; PMID: 14967113

2. McMurray J, Hart W, Rhodes G. An evaluation of the cost of heart failure to the National Health Service in the UK. Br J Med Econ. 1993;6:91-8. 
3. Anguita Sánchez M, Crespo Leiro MG, De Teresa Galván E, Jiménez Navarro M, Alonso-Lupón L, Muñiz García J. Prevalencia de la insuficiencia cardiaca en la población general española mayor de 45 años: Estudio PRICE. Rev Esp Cardiol. 2008;61(10):1041-9. DOI: 10.1157/13126044

4. Datos de población en el País Vasco a través de los censos de 1981 a 2016 (página Web) [consultada 31 mayo de 2019]. Disponible en: www.eustat.eus

5. Lee DS, Austin PC, Stukel TA, Alter DA, Chong A. Parker JD, et al. "Dose-dependent" Impact of Recurrent Cardiac Events on Mortality in Patients with Heart Failure. Am J Med. 2009;122(2):162169.e1. DOI: 10.1016/j.amjmed.2008.08.026; PMID: 19100960

6. Banegas JR, Rodriguez-Artalejo F. Insuficiencia cardiaca e instrumentos para medir la calidad de vida [Editorial]. Rev Esp Cardiol 2008;61 (3):233-5. DOI: 10.1157/13116649

7. Llorens Soriano P, Miró O, Martín-Sánchez FJ, Herrero Puente P, Jacob Rodríguez J, Gil V, et al; Grupo ICA-SEMES. Manejo de la insuficiencia cardiaca aguda en los servicios de urgencias, emergencias y unidades adscritas. Documento de consenso del Grupo de Insuficiencia Cardiaca Aguda de la Sociedad Española de Medicina de Urgencias y Emergencias (ICA-SEMES). Emergencias. 2011;23(2):119-39.

8. Collins SP, Pang PS. ACUTE Heart Failure Risk Stratification: A Step Closer to the Holy Grail? Circulation. 2019;139(9):1157-61. DOI: 10.1161/CIRCULATION AHA.118.038472

9. Shepperd S, Doll H, Angus RM, Clarke MJ, lliffe S, Kalra L, et al. Hospital at home admission avoidance. Cochrane Database Syst Rev. 2008;(4):CD007491. DOI: 10.1002/14651858. CD007491; PMID: 18843751

10. Tibaldi V, Isaia G, Scarafiotti C, Gariglio F, Zanocchi M, Bo M, et al. Hospital at home for elderly patients with acute decompensation of chronic heart failure: a prospective randomized controIled trial. Arch Intern Med. 2009;169(17):1569-75. DOI: 10.1001/archinternmed.2009.267; PMID: 19786675

11. Mendoza H, Martin MJ, Garcia-Soleto A, Arós F, Aizpuru F, Regalado de los Cobos J, et al. "Hospital at home" care model as an effective alternative in the management of decompensated chronic heart failure. Eur J Heart Fail. 2009;11(12):1208-13. DOI: 10.1093/eurjhf/hfp143; PMID: 19875400

12. Lee DS, Austin PC, Rouleau JL, Liu PP, Naimark D, Tu JV. Predicting mortality among patients hospitalized for heart failure: derivation and validation of a clinical model. JAMA. 2003;290(19):2581-7. DOI: 10.1001/jama.290.19.2581; PMID: 14625335

13. Regalado de los Cobos, Delgado Vicente M, Oceja Barrutieta E, Matía Sanz M, Apraiz Garmendia L, de Juan Rodríguez M. Evitar ingresos hospitalarios por insuficiencia cardiaca: estimación a partir de una experiencia. $12^{\circ}$ Congreso Nacional Hospitalización a Domicilio. Burgos. 5-7 de Junio de 2014.

14. Formiga F, Chivite D, Casas S, Manito N, Pujol R. Valoración funcional en pacientes ancianos ingresados por insuficiencia cardiaca. Rev Esp Cardiol. 2006;59(7):740-2. DOI: 10.1157/13091377

15. Martín-Sánchez FJ, Gil V, Llorens P, Herrero P, Jacob J, Fernandez C, et al; Acute Heart Failure Working Group of the Spanish Society of Emergency Medicine Investigation Group. Barthel Index-Enhanced Feedback for Effective Cardiac Treatment (BI-EFFECT) Study: contribution of the Barthel Index to the Heart Failure Risk Scoring System model in elderly adults with acute heart failure in the emergency department. J Am Geriatr Soc. 2012;60(3):493-8. DOI:10.1111/j.15325415.2011.03845.x; PMID: 22329408

16. Miró O, Llorens P, Martín-Sänchez FJ, Herrero P, Jacob J, Pérez-Durá MJ, et al. Predicting the risk of reattendance for acute heart failure patients discharged from Spanish Emergen- 
ce Department observation units. Eur J Emerg Med. 2010;17(4):197-202. DOI: 10.1097/ MEJ.0b013e32832f7666; PMID: 20215973

17. Muzarelli S, Leibundgut G, Maeder MT, Rickli H, Handschin R, Gutmann M, et al. Predictors of early readmission or death in elderly patients with heart failure. Am Heart J. 2010;160(2):308-14. DOI: 10.1016/j.ahj.2010.05.007; PMID: 20691837

18. Philbin EF, Di Salvo TG. Prediction of hospital readmission for heart failure: development of a simple risk score based on administrative data. J Am Coll Cardiol. 1999;33(6):1560-6. PMID: 10334424

19. Jiménez S, Aguilo S, Gil V, Antolín A, Prieto S, Bragulat E, et al. Los factores psicosociales determinan la aceptación de la hospitalización a domicilio directamente desde el sevicio de urgencias. Gac Sanit. 2010;24(4):303-8. DOI: 10.1016/j.gaceta.2010.03.012; PMID: 20663592 